\title{
The physical health of people with severe mental illness in Bangladesh and Pakistan: A cross-sectional survey
}

\author{
Gerardo Antonio Zavala ( g.zavala@york.ac.uk) \\ ARK Foundation Bangladesh https://orcid.org/0000-0002-9825-8725 \\ Asiful Haidar \\ ARK Foundation Bangladesh \\ Krishna Prasad-Muliyala \\ NIMHANS: National Institute of Mental Health and Neuro Sciences \\ Faiza Aslam \\ IOP: Institute of Psychiatry \\ Rumana Huque \\ Arkin Foundation Bangladesh \\ Humaira Khalid \\ IOP: Institute of Psychiatry \\ Pratima Murthy \\ NIMHANS: National Institute of Mental Health and Neuro Sciences \\ Asad T Nizami \\ IOP: Institute of Psychiatry \\ Sukanya Rajan \\ NIMHANS: National Institute of Mental Health and Neuro Sciences \\ David Shiers \\ University of Manchester \\ Najma Siddiqi \\ University of York \\ Kamran Siddiqi \\ University of York \\ Jan R Boehnke \\ University of Dundee
}

\section{Research article}

Keywords: Physical health conditions, Health risk behaviour, Multimorbidity, Comorbidity, Severe mental illness, South Asia, Low and middleincome countries

Posted Date: April 20th, 2021

DOI: https://doi.org/10.21203/rs.3.rs-429162/v1

License: (c) (i) This work is licensed under a Creative Commons Attribution 4.0 International License. Read Full License 


\section{Abstract}

Background

People with severe mental illness (SMI) die earlier than the general population, primarily due to physical disorders. There is limited information on physical illnesses and health-risk behaviours in people with SMI in low and middle-income countries.

Methods

We conducted a cross-sectional survey in adults with SMI attending specialist mental health services in Bangladesh and Pakistan. Data were collected on non-communicable diseases (NCDs), their risk factors, health-risk behaviours, treatments and health risk modification advice (using questions from the WHO STEPwise approach to Surveillance of NCDs (STEPS)) and on common mental disorders, health-related quality of life and infectious diseases. We performed a descriptive analysis, and compared weighted prevalence for these variables in our survey with prevalence for the general population in the STEPS reports from Bangladesh and Pakistan.

Results

We recruited 2,344 participants with bipolar disorder (36.7\%), non-affective psychosis (42.2\%), and depression with psychosis (21.1\%). Eight percent had diabetes, $24.7 \%$ hypertension and $3.1 \%$ tuberculosis. $43.4 \%$ were overweight or obese, and half had hypercholesterolemia. Most participants with diabetes, hypertension and hypercholesterolemia were previously undiagnosed; of those diagnosed only around half were receiving treatment. Fifty-four percent of men and $17.2 \%$ of women used tobacco; $46.9 \%$ and $87.1 \%$ did not meet WHO recommendations for physical activity and fruit and vegetable intake respectively. Compared with the general population, people with SMI were more likely to have diabetes (O.R. $=1.56,95 \%$ C.I $=1.30$ to 1.88 Bangladesh), hypercholesterolemia (O.R. $=2.35,95 \%$ C.I. $=2.08$ to 2.65 Bangladesh) and overweight or obesity (O.R. $=1.97,95 \%$ C.I. $=1.75$ to 2.22 Bangladesh; O.R. $=1.61,95 \%$ C.I. $=1.40$ to 1.86 Pakistan). They were less likely to receive tobacco cessation (O.R. $=0.33,95 \%$ C.I. $=0.26$ to 0.42 Bangladesh; $0 . R .=0.42,95 \%$ C.I $=0.31$ to 0.55 Pakistan), and weight management advice (O.R. $=0.51,95 \%$ C.I. $=0.41$ to 0.63 Bangladesh; and O.R. $=0.65,95 \%$ C.I. $=0.51$ to 0.82 Pakistan).

Conclusion

Despite the high prevalence we found significant gaps in detection, prevention and treatment of NCDs and their risk factors in people with SMI.

Registration

ISRCTN88485933; https://doi.org/10.1186/ISRCTN88485933

\section{Introduction}

Severe mental illnesses (SMI) are conditions such as schizophrenia and bipolar disorder that are debilitating, persistent and associated with serious functional impairment. People with SMI die on average 10-20 years earlier than the general population, and this 'mortality gap' is widening.[1] Although suicide accounts for $15 \%$ of deaths, an estimated $80 \%$ of the observed premature mortality is attributable to physical disorders (physical multimorbidity), most commonly due to non-communicable diseases (NCDs).[2]

The excess disease burden from physical multimorbidity in people with SMI may be explained by a combination of factors associated with these mental disorders including clustering of, and predisposition to health-risk behaviours (e.g. tobacco and alcohol use, lack of physical activity, and poor diet), side effects of medication, social determinants of poor health (e.g. stigma, and poverty) and barriers to accessing healthcare.[3] Our current understanding of the distribution and determinants of physical multimorbidity in people with SMI is based mostly on evidence from high-income countries. A few small studies from low- and middle-income countries (LMICs) show similar patterns, but with an even shorter life expectancy and higher mortality for people with SMI.[4, 5] These studies indicate that physical multimorbidity in SMI may be at least as much of a challenge in LMICs as in high-income countries.[1]

In South Asia, the prevalence of both mental disorders and NCDs has been increasing rapidly.[6, 7] This increase is coupled with limited access to essential health services and a widespread neglect of the physical health needs of people with SMI by policy makers and healthcare services.[8] The overall burden of disease due to physical multimorbidity in this population is, therefore, likely to be high and is set to rise further, with a corresponding increase in within-country and global health inequalities. Despite these concerns, there is a lack of empirical studies originating in South Asia on the distribution and determinants of physical multimorbidity in people with SMI.[9] 
Addressing multimorbidity in LMICs is a global priority, recognised in global policies to help achieve the Sustainable Development Goals.[10] A detailed understanding of the prevalence of physical multimorbidity and current access to health advice and treatments for physical disorders in people with SMI in LMICs can inform appropriate service provision and contribute to achieving these goals.

\section{Methods}

\section{Aims}

1) estimate the prevalence of physical health conditions and health risk behaviours, 2) assess access to physical healthcare and health-risk modification advice, and 3) compare findings with those of the general population. for people with SMI in Bangladesh, and Pakistan.

\section{Design and settings}

We conducted a cross-sectional survey of patients with a clinical diagnosis of SMI recruited at two national specialist mental health institutions in South Asia, the National Institute of Mental Health (NIMH) in Dhaka, Bangladesh; and Institute of Psychiatry (IOP) Rawalpindi, Pakistan. We originally planned to include a third site- the National Institute of Mental Health and Neurosciences, Bangalore, India, However, due to a delay in obtaining all requisite approvals, data collection could not be initiated before COVID-19 pandemic. Further details of the methods are reported in the published protocol,[11] and summarised below.

\section{Sample size}

We aimed to build as large a sample as possible within the resources available over the study period, with an initial target of 1,500 participants at each site. As an indicative example of precision to address some of the key research questions, we used the example of diabetes. For investigating the prevalence of type-2 diabetes, assuming a prevalence estimate of $10 \%, 857$ participants per country would provide a precision of $\pm 2 \%$ (95\% confidence interval).

\section{Eligibility}

Consenting adults (18 years and over) with a clinical diagnosis of SMI defined by the International Classification of Disease 10th revision (ICD-10) as schizophrenia, schizotypal and delusional disorders (F20-F29); bipolar affective disorder (F30, F31); and severe depression with psychotic symptoms (F32.3, F33.3), and able to provide informed consent, as assessed by the treating clinician, were eligible.

\section{Confirmation of SMI diagnosis}

To increase standardisation across sites and alignment with other studies, each SMI diagnosis was confirmed by trained researchers using the Mini-international neuropsychiatric interview (MINI) version 6.0.[12] The MINI is a short diagnostic structured interview for mental disorders, designed to allow administration by non-specialists.

\section{Recruitment of participants}

We used stratified random sampling to recruit a sample comprising $80 \%$ outpatients and $20 \%$ inpatients, reflecting the service case mix.[11]

\section{Patient and public involvement}

A community panel comprising patients, caregivers and advocacy group members ensured community, patient, and public involvement. The panel reviewed and piloted the planned survey questionnaire and advised on its feasibility.

\section{Data collection}

We conducted a face-to-face survey using tablets (Qualtrics, Provo, UT) to collect information about physical disorders, mental health, health risk behaviours, health-related quality of life, health-risk behaviour advice and healthcare utilisation using, wherever available, validated instruments as described below. The survey was translated into Urdu and Bangla. Interviewers (including males and females to 
accommodate participant preference) used regional dialects where required, consistent with usual clinical practice in these settings. Data were collected between July 2019 and March 2020, when recruitment was halted due to the COVID-19 outbreak.

\section{STEPwise approach to surveillance (STEPS)}

We used the WHO STEPwise approach to Surveillance of NCDs (STEPS) instrument Version 3.2 to collect information about NCDs, associated risk factors and behaviours, access to physical healthcare and health-risk modification advice.[13] STEPS is an international standardised tool that has already been translated, used and validated in the general population in Bangladesh and Pakistan, and therefore allows comparisons with the general population within and between countries.[14,15] The STEPS survey includes the use of show-cards with culturally relevant examples used to aid respondents in classifying health-risk behaviours. Categorisation of health conditions and risk behaviours followed the WHO guidelines.[16]

The STEPS module for NCDs was used to ask participants about medically-diagnosed type-2 diabetes, raised blood pressure, heart disease, and hypercholesterolemia, and treatments advised by a health worker for these conditions (such as medication and dietary, weight management, smoking cessation, or physical activity advice). Questions about lung disease, hepatitis B, C, syphilis, tuberculosis (TB) and human immunodeficiency virus (HIV) (which are not part of the STEPS survey) were asked in the same format as for the other chronic physical conditions.

Health-risk behaviours

Current or past use of smoked or smokeless tobacco was recorded.[16] The alcohol module was used to categorise participants into lifetime abstainers, abstainers in the past 12 months and current users of alcohol;[16] and the diet module was used to record the number of days that respondents consumed fruit and vegetables in a typical week, the number of servings consumed on average per day, and adherence to the WHO recommendations of at least 5 fruits and vegetables per day.[17] The physical activity module was used to record activity for transport purposes (e.g. walking, cycling), vigorous and moderate activity at work, and vigorous, moderate activity in leisure time, time spent sitting and adherence to the WHO recommendations of $<600$ metabolic equivalents minutes/week.[18] In addition, risk behaviours related to sexually transmitted diseases, including multiple sexual partners, unprotected sexual contact, and use of injectable drugs, were assessed using three questions adapted from the 10-item HIV risk Screening Instrument.[19, 20]

Physical measurements

Blood pressure (BP) was taken using an automated blood pressure measuring instrument (OMRON $®$ ) following instructions in the WHO STEPs surveillance manual); the average of the second and third readings was used for analysis.[16] High blood pressure was defined as a measurement of $>140 / 90 \mathrm{mmHg}$.[16]

Height, weight, and waist circumference were measured for all participants except pregnant women. All measurements were taken in duplicate and the average of the two values was calculated, following the protocols set out in the WHO STEPS surveillance manual.[16] We calculated the Body Mass Index (BMI) and classified participants using the WHO classification, namely underweight (BML $<18.49$ ), normal weight (BMl= 18.5-24.9), overweight (BMI= 25-29.9), obesity (BMl $\geq 30)$. Abdominal obesity was defined as a waist circumference of $(\geq 94$ $\mathrm{cm})$ for males and $(\geq 80 \mathrm{~cm})$ for females.[16]

Mental health

In addition to administering the MINI, we collected information relevant to the SMI diagnosis, including duration of illness and type and duration of treatments. The Patient Health Questionnaire (PHQ-9) was used to measure the severity of depressive symptoms,[21] and the Generalized Anxiety Disorder-7 (GAD-7) for severity of anxiety symptoms.[22]

Health-Related Quality of life

The EQ-5D-5L was used to measure health-related quality of life.[23] We used the Urdu and Bangla validated versions, provided by EuroQol.

Blood Tests

A blood sample was taken from consenting participants for: haemoglobin, glycated haemoglobin (HbA1c), lipid profile, thyroid function tests, liver function tests and creatinine. The cut off for high $\mathrm{HbA1c}$ was according to the WHO definition $\geq 6.5 \%$.[24] The prevalence of high total triglycerides was defined as $\geq 180 \mathrm{mg} / \mathrm{dl}$,[25] high serum cholesterol was defined as $L D L \geq 100 \mathrm{mg} / \mathrm{dl}$.[25] Anaemia was defined according as haemoglobin $\leq 13 \mathrm{~g} / \mathrm{dl}$ for males and $\leq 12 \mathrm{~g} / \mathrm{dl}$ for females.[26] 


\section{Statistical analysis}

Quantitative data were summarised using descriptive statistics with means, standard deviation and $95 \%$ confidence interval for continuous data and counts, percentages and $95 \%$ confidence interval for categorical data. Overall, and by site we described the:

1) prevalence of chronic physical conditions.

2) prevalence of risk factors (obesity, hypertension, hypercholesterolemia) and risk behaviours (poor diet, physical inactivity, tobacco and alcohol use).

3) severity of common mental disorder symptoms (anxiety, depression) and health-related quality of life.

4) access to treatment for physical conditions and health risk modification advice

To compare our findings with those in the latest STEPS reports from Bangladesh[15] and Pakistan,[14] we calculated weights by comparing the sex and age distribution reported in these STEPS surveys with the distribution in our data. Weights were applied using the complex sample module in SPSS 26.0 and we calculated the odds of people with SMI of having a NCD, related risk factors, engaging in health risk behaviours and being screened, treated and receiving risk modification advice compared with the STEPS survey participants in Bangladesh and Pakistan.[14, 15] Results were presented as odds ratios from cross-tabulations of STEPS and weighted survey data. Significance levels were adjusted via Bonferroni correction for multiple hypothesis testing (adjusted level $p<0.006$ ).[27]

\section{Results}

Between July 2019 and March 2020, 2,757 people with SMI were approached in the two sites and 2,344 (85.0\%) participated in the survey (1422 in Bangladesh and 922 in Pakistan). Out of those who did not participate, 368 were ineligible, 36 refused and 9 left before completing the survey (Fig. 1).

Participant characteristics are shown in Table 1. On average, $60.5 \%$ of the sample was male, and the mean age was 34.2 years - the Bangladesh cohort was younger (31.5 years, 95\%C.I. $=30.9$ to 32.0 ) than the cohort in Pakistan (38.3 years, 95\%C.I. $=37.5$ to 39.0 ). Almost one third (32.0\%) were unemployed, with a higher proportion in Bangladesh (39.0\%, 95\%C.I. $=35.9$ to 42.3$)$ than Pakistan $(21.2 \%, 95 \%$ C.I. $=18.1$ to 24.3). The majority (54.9\%) were educated only up to, or less than primary school level. One third $(33.3 \%, 95 \%$ C.I. $=30.3$ to 36.5$)$ of participants in Bangladesh had completed secondary school or higher education, while almost two thirds $(63.1 \%, 95 \%$ C.I. $=59.2$ to 66.9$)$ in Pakistan had achieved this level. 
Table 1

General characteristics of the sample

\begin{tabular}{|c|c|c|c|}
\hline & Bangladesh $(N=1422)$ & Pakistan ( $\mathrm{N}=922)$ & Overall $(\mathrm{N}=2344)$ \\
\hline & n (\%) [95\% C.I.] & n (\%) [95\% C.I.] & n (\%) [95\% C.I.] \\
\hline \multicolumn{4}{|l|}{ General characteristics } \\
\hline Gender (Female) & $558(39.2)[36.7-41.8]$ & $367(39.8)$ [36.7-42.9] & 925 (39.5) [37.5-41.5] \\
\hline Age (years)* & $31.5(10.8)[30.9-32.0]$ & $38.3(12.3)$ [37.5-39.0] & $34.2(11.9)[33.7-34.6]$ \\
\hline \multicolumn{4}{|l|}{ Education } \\
\hline No formal education & 147 (10.3) [8.5-12.5] & $184(20.0)[16.9-23.6]$ & $331(14.1)$ [12.8-15.6] \\
\hline Primary & $801(56.3)[53.1-59.5]$ & $156(16.9)[14.1-20.2]$ & 957 (40.8) [39.0-42.7] \\
\hline Secondary/higher & $474(33.3)[30.3-36.5]$ & $582(63.1)[59.2-66.9]$ & $1056(45.1)[43.1-47.0]$ \\
\hline $\begin{array}{l}\text { Monthly household income over last year } \\
\text { (USD)* }\end{array}$ & $\begin{array}{l}223.0(359.3)[207.1- \\
245.2]\end{array}$ & $\begin{array}{l}203.4(200.3)[190.7- \\
216.9]\end{array}$ & $\begin{array}{l}215.4(307.0)[202.9- \\
227.8]\end{array}$ \\
\hline Cement/Concrete roof & $384(27.0)$ [24.7-29.2] & 719 (78.0) [75.4-80.5] & $384(27.0)[24.8-29.4]$ \\
\hline Electricity in the household & 1388 (97.6) [96.7-98.3] & $912(98.9)$ [98.2-99.6] & 2300 (98.1) [97.5-98.6] \\
\hline Flush toilet in the household & $811(57.0)[54.4-59.4]$ & $896(97.2)$ [96.1-98.2] & 1707 (72.8) [71.2-74.4] \\
\hline \multicolumn{4}{|l|}{ Occupation‡ } \\
\hline Non-government employee & 145 (10.2) [7.5-13.6] & $265(28.7)[23.5-34.6]$ & 410 (17.5) [16.0-19.0] \\
\hline Government employee & $15(1.1)[0.6-1.7]$ & $39(4.2)[3.1-5.7]$ & $54(2.3)[1.8-3.0]$ \\
\hline Self-employed & $243(17.1)[13.7-21.2]$ & $53(5.7)[3.4-9.3]$ & 296 (12.6) [11.4-14.0] \\
\hline Student & 109 (7.7) [5.4-10.7] & $19(2.1)[0.9-4.6]$ & $128(5.5)[4.6-6.5]$ \\
\hline Homemaker & $336(23.6)[19.7-28.1]$ & $318(34.5)[28.9-40.1]$ & $654(27.9)[26.1-29.7]$ \\
\hline Unemployed (able to work) & 276 (19.4) [15.7-23.6] & $174(18.9)[14.5-24.1]$ & $450(19.2)[17.7-20.8]$ \\
\hline Unemployed (unable to work) & 279 (19.6) [15.9-23.8] & $21(2.3)[1.0-5.0]$ & $300(12.8)[11.5-14.2]$ \\
\hline Did not wish to answer & $0(0.0)$ & $12(1.3)[0.4-3.6]$ & $12(0.5)[0.3-0.9]$ \\
\hline Currently married/living with partner & $782(55.0)[52.1-57.5]$ & $514(55.7)[52.7-59.0]$ & $1296(55.3)[53.3-57.3]$ \\
\hline \multicolumn{4}{|l|}{ Severe mental illness (MINI) } \\
\hline Bipolar disorder & $468(32.9)[34.3-39.2]$ & $392(42.5)[38.6-46.5]$ & $860(36.7)[34.3-39.2]$ \\
\hline Non-affective psychosis & $882(62.0)[58.8-65.1]$ & 108 (11.7) [9.4-14.6] & $990(42.2)[39.7-44.7]$ \\
\hline $\begin{array}{l}\text { Major depressive disorder with psychotic } \\
\text { features }\end{array}$ & $72(5.1)[3.8-6.7]$ & $422(45.8)[41.8-49.8]$ & $494(21.1)[19.1-23.2]$ \\
\hline \multicolumn{4}{|l|}{ Type or setting of patients } \\
\hline Inpatient & $313(22.0)[19.9-24.3]$ & $64(6.9)[5.4-8.6]$ & $377(16.1)[14.7-17.6]$ \\
\hline Outpatient & 1109 (78.0) [75.7-80.1] & $858(93.1)$ [91.4-94.6] & $1967(83.9)[82.4-85.3]$ \\
\hline \multicolumn{4}{|l|}{ Duration of SMI } \\
\hline Less than 2 years & $410(28.8)[25.8-23.6]$ & $187(20.3)[16.5-24.6]$ & $597(25.5)$ [23.8-27.3] \\
\hline 3 to 5 years & $430(30.2)[26.6-34.1]$ & $211(22.9)[18.9-27.4]$ & $641(27.3)[25.6-29.2]$ \\
\hline 6 to 10 years & 319 (22.4) [19.1-26.0] & $228(24.7)[20.6-29.4]$ & $547(23.3)[21.7-25.1]$ \\
\hline More than 10 years & 259 (18.2) [15.2-21.6] & $296(32.1)[27.6-37.0]$ & 555 (23.7) [22.0-25.4] \\
\hline Do not know or do not remember & $4(0.3)[0.1-0.6]$ & $0(0.0)$ & $4(0.2)[0.1-0.5]$ \\
\hline
\end{tabular}




\begin{tabular}{|c|c|c|c|}
\hline & Bangladesh $(N=1422)$ & Pakistan ( $\mathrm{N}=922)$ & Overall $(\mathrm{N}=2344)$ \\
\hline On antipsychotic medication & 1385 (97.4) [96.6-98.2] & $882(95.7)$ [94.3-96.9] & 2267 (96.7) [95.9-97.4] \\
\hline \multicolumn{4}{|l|}{ Mental health } \\
\hline \multicolumn{4}{|l|}{ Severity of depressive symptoms } \\
\hline PHQ9 score* & $10.6(4.6)[10.4-10.8]$ & $13.4(6.9)[13.0-13.9]$ & $11.7(5.8)[11.5-11.9]$ \\
\hline None- or minimal (0 to 4 ) & $117(8.2)[6.6-10.2]$ & $111(12.0)[9.6-14.9]$ & $228(9.7)[8.6-11.0]$ \\
\hline Mild (5 to 9) & $470(33.1)[30.7-35.5]$ & $170(18.4)[15.5-21.7]$ & $640(27.3)[25.6-29.1]$ \\
\hline Moderate or Severe (more than 10) & $835(58.6)[55.5-61.9]$ & $641(69.5)[66.5-72.4]$ & $1476(63.0)[61.0-64.9]$ \\
\hline \multicolumn{4}{|l|}{ Severity of anxiety symptoms } \\
\hline GAD7 score* & $8.0(3.9)[7.8-8.2]$ & $10.4(5.1)[9.1-10.8]$ & $9.0(4.5)[8.8-9.2]$ \\
\hline None- or minimal ( 0 to 4$)$ & $261(18.4)[15.9-20.9]$ & $116(12.6)[10.1-15.5]$ & $377(16.1)[14.7-17.6]$ \\
\hline Mild (5 to 9 ) & $671(47.2)[43.9-50.4]$ & $292(31.7)[28.0-35.6]$ & $963(41.1)[39.1-43.1]$ \\
\hline Moderate or Severe (10 to 21$)$ & $490(34.4)[31.4-37.6]$ & $514(55.8)[51.7-59.7]$ & $1004(42.8)[40.9-44.8]$ \\
\hline \multicolumn{4}{|l|}{ Health related quality of life } \\
\hline Visual analog scale * & $69.9(13.6)[69.2-70.5]$ & $55.1(28.0)[53.3-57.0]$ & $64.0(21.7)[63.2-64.9]$ \\
\hline Mobility & $435(30.6)[28.2-33.0]$ & $530(57.5)[54.4-60.8]$ & $965(41.2)[39.3-43.1]$ \\
\hline Self-Care & $486(34.2)[31.8-36.7]$ & 448 (48.6) [45.5-51.7] & $934(39.8)[37.9-41.8]$ \\
\hline Usual Activities & $640(45.0)[42.4-47.6]$ & $536(58.1)[54.8-61.4]$ & $1176(50.2)[48.2-52.2]$ \\
\hline Pain/Discomfort & $934(65.7)[63.2-68.1]$ & 721 (78.2) [75.5-80.8] & 1655 (70.6) [68.7-72.4] \\
\hline Anxiety/Depression & $1286(90.4)$ [89.0-91.9] & 694 (75.3) [72.4-78.1] & 1980 (84.5) [83.0-85.9] \\
\hline
\end{tabular}

\section{SMI, anxiety and depressive symptoms and health-related quality of life}

The most common SMI diagnosis was non-affective psychosis (42.2\%), followed by bipolar disorder (36.7\%) and depression with psychotic symptoms (21.1\%). Non-affective psychosis was the most common diagnosis in Bangladesh $(62.0 \%, 95 \%$ C.I. $=58.8$ to 65.1$)$ and depression with psychotic symptoms $(45.8 \%, 95 \%$ C.I. $=41.8$ to 49.8$)$ the most common in Pakistan. $96.7 \%$ of participants were on antipsychotic medication.

A significant proportion of participants reported depressive (63.0\%) and anxiety (42.8\%) symptoms in the 'moderate or severe' category, higher in Pakistan $(69.5 \%, 95 \%$ C.I. $=66.5$ to 72.4$)$ than Bangladesh $(58.6 \%, 95 \%$ C.I. $=55.5$ to 61.9$)$. Similarly, there was a higher proportion reporting 'moderate or severe' anxiety symptoms in Pakistan ( $55.8 \%, 95 \%$ C.I. $=51.7$ to 59.7$)$ than Bangladesh ( $34.4 \%, 95 \%$ C.I. $=31.4$ to 37.6$)$.

The mean EQ-5D (health-related quality of life) visual analogue scale (0-100) score was 64.0 overall, and higher for Bangladesh (69.9, $95 \%$ C.I. $=69.2$ to 70.5$)$ than Pakistan $(55.1,95 \%$ C.I. $=53.3$ to 57.0$)$. One half of participants reported problems in carrying out their usual activities; and around seventy percent reported pain/discomfort (Table 1).

\section{Physical disorders, risk factors, health risk behaviours and healthcare}

Tables 2 and 3 summarise findings for the prevalence of physical disorders, risk factors and healthcare use. 
Table 2

Non-communicable and communicable diseases and health risk behaviours in people with SMI

\begin{tabular}{|c|c|c|c|}
\hline & $\begin{array}{l}\text { Bangladesh }(\mathrm{N}= \\
1422)\end{array}$ & $\begin{array}{l}\text { Pakistan }(\mathrm{N}= \\
922)\end{array}$ & Overall $(\mathrm{N}=2344)$ \\
\hline & n (\%) [95\% C.I.] & n (\%) [95\% C.I.] & n (\%) [95\% C.I.] \\
\hline \multicolumn{4}{|l|}{ Non communicable diseases (NCDs) } \\
\hline $\begin{array}{l}\text { Type-2 diabetes diagnosed by HbA1c test ( } \geq 6.50 \% \text { ) or self-report of } \\
\text { clinician diagnosis } \S\end{array}$ & $\begin{array}{l}111(8.1)[6.8- \\
9.6]\end{array}$ & $72(8.0)[6.4-9.9]$ & $183(8.1)[7.0-9.3]$ \\
\hline Type-2 diabetes (self-report of clinician diagnosis only) & 38 (2.7) [1.9-3.7] & $55(6.0)[4.6-7.7]$ & $93(4.0)[3.3-4.8]$ \\
\hline Heart disease (angina or a stroke) (self-report of clinician diagnosis) & 19 (1.3) [0.8-2.0] & $65(7.0)[5.5-8.6]$ & $84(3.6)[2.9-4.4]$ \\
\hline Lung condition (self-report of clinician diagnosis) $\ddagger$ & - & - & $31(1.3)[0.9-1.9]$ \\
\hline \multicolumn{4}{|l|}{ NCDs risk factors } \\
\hline $\begin{array}{l}\text { High blood pressure (blood pressure } \geq 140 / 90 \mathrm{mmHg} \text { ) or self-report of } \\
\text { clinician diagnosis } \S\end{array}$ & $\begin{array}{l}235(16.5)[14.6- \\
18.5]\end{array}$ & $\begin{array}{l}344(37.4)[34.2- \\
40.4]\end{array}$ & $\begin{array}{l}579(24.7)[23.1- \\
26.4]\end{array}$ \\
\hline High blood pressure (self-report of clinician diagnosis only) & $82(5.8)[4.7-7.1]$ & $\begin{array}{l}240(26.0)[23.3- \\
29.0]\end{array}$ & $\begin{array}{l}322(13.7)[12.5- \\
15.1]\end{array}$ \\
\hline Hypercholesterolemia (tested) plus self-report of clinician diagnosis $\S$ & $\begin{array}{l}608(44.8)[42.2- \\
47.5]\end{array}$ & $\begin{array}{l}497(55.8)[52.5- \\
59.1]\end{array}$ & $\begin{array}{l}1105(49.2) \\
{[47.1-51.2]}\end{array}$ \\
\hline Hypercholesterolemia (self-report of clinician diagnosis only) & $13(0.9)[0.5-1.6]$ & $45(4.9)[3.7-6.5]$ & $58(2.5)[1.9-3.2]$ \\
\hline Body Mass Index $\left(\mathrm{Kg} / \mathrm{m}^{2}\right)$ * & $\begin{array}{l}24.0(4.6)[23.8- \\
24.3]\end{array}$ & $\begin{array}{l}26.4(9.5)[25.9- \\
27.1]\end{array}$ & $\begin{array}{l}25.0(7.0)[24.7- \\
25.3]\end{array}$ \\
\hline \multicolumn{4}{|l|}{ Body Mass Index categories } \\
\hline Underweight $(<18.5)$ & $\begin{array}{l}123(8.6)[6.8- \\
10.9]\end{array}$ & $\begin{array}{l}78(8.5)[6.2- \\
12.5]\end{array}$ & $201(8.6)[7.5-9.8]$ \\
\hline Normal weight (18.5 to 24.9) & $\begin{array}{l}777(54.8)[51.4- \\
58.5]\end{array}$ & $\begin{array}{l}343(37.5)[33.2- \\
42.1]\end{array}$ & $\begin{array}{l}1120(48.0) \\
{[46.0-50.0]}\end{array}$ \\
\hline Overweight (25 to 29.9 ) & $\begin{array}{l}379(26.7)[23.5- \\
30.1]\end{array}$ & $\begin{array}{l}284(31.1)[26.9- \\
35.5]\end{array}$ & $\begin{array}{l}663(28.4)[26.6- \\
30.3]\end{array}$ \\
\hline Obese $(\geq 30)$ & $\begin{array}{l}140(9.9)[7.8- \\
12.3]\end{array}$ & $\begin{array}{l}209(22.9)[19.2- \\
26.9]\end{array}$ & $\begin{array}{l}349(15.0)[13.6- \\
16.4]\end{array}$ \\
\hline Waist circumference $(\mathrm{cm})^{\star}$ & $\begin{array}{l}84.1(11.6) \text { [83.5- } \\
84.6]\end{array}$ & $\begin{array}{l}92.4(18.4) \\
{[91.2-93.6]}\end{array}$ & $\begin{array}{l}87.3(15.2)[86.7- \\
87.9]\end{array}$ \\
\hline High waist circumference women $(\geq 80 \mathrm{~cm})$ & $\begin{array}{l}385(69.4)[65.8- \\
73.5]\end{array}$ & $\begin{array}{l}286(79.4)[75.5- \\
83.7]\end{array}$ & $\begin{array}{l}671(73.3)[70.4- \\
76.1]\end{array}$ \\
\hline High waist circumference men $(\geq 94 \mathrm{~cm})$ & $\begin{array}{l}127(14.7)[12.5- \\
17.1]\end{array}$ & $\begin{array}{l}235(42.9)[38.8- \\
47.0]\end{array}$ & $\begin{array}{l}362(25.6)[23.5- \\
27.9]\end{array}$ \\
\hline \multicolumn{4}{|l|}{ Communicable diseases } \\
\hline Hepatitis B or hepatitis C (self-report of clinician diagnosis) $\ddagger$ & - & - & 61 (2.6) [2.0-3.3] \\
\hline Tuberculosis (self-report of clinician diagnosis) & $18(1.3)[0.7-1.9]$ & $54(5.9)[4.4-7.4]$ & $72(3.1)[2.4-3.8]$ \\
\hline HIV (self-report of clinician diagnosis only) $\ddagger$ & - & - & - \\
\hline \multicolumn{4}{|l|}{ Physical activity } \\
\hline Time spent on vigorous physical activity (min/day) * & $\begin{array}{l}17.7(69.1)[14.3- \\
21.3]\end{array}$ & $\begin{array}{l}24.4(87.0) \\
{[18.8-30.2]}\end{array}$ & $\begin{array}{l}20.3(76.7)[17.2- \\
23.4]\end{array}$ \\
\hline Vigorous physical activity (>0 min/day) & $\begin{array}{l}169(11.9) \text { [10.3- } \\
13.6]\end{array}$ & $\begin{array}{l}149(16.2)[13.8- \\
18.8]\end{array}$ & $\begin{array}{l}318(13.6)[12.2- \\
15.0]\end{array}$ \\
\hline Time spent on moderate physical activity (min/day) * & $\begin{array}{l}30.9(65.8)[27.4- \\
34.4]\end{array}$ & $\begin{array}{l}24.9(83.4)[20.0- \\
30.6]\end{array}$ & $\begin{array}{l}28.5(73.2) \text { [25.6- } \\
31.5]\end{array}$ \\
\hline
\end{tabular}




\begin{tabular}{|c|c|c|c|}
\hline & $\begin{array}{l}\text { Bangladesh }(\mathrm{N}= \\
1422)\end{array}$ & $\begin{array}{l}\text { Pakistan }(\mathrm{N}= \\
\text { 922) }\end{array}$ & Overall $(\mathrm{N}=2344)$ \\
\hline Moderate physical activity (>0 min/day) & $\begin{array}{l}560(39.4)[36.8- \\
42.0]\end{array}$ & $\begin{array}{l}236(25.6)[22.8- \\
28.5]\end{array}$ & $\begin{array}{l}796(34.0)[32.1- \\
35.9]\end{array}$ \\
\hline Time spent walking/cycling (min/day)* & $\begin{array}{l}33.2(47.1)[30.7- \\
35.6]\end{array}$ & $\begin{array}{l}29.5(81.2) \\
{[24.8-35.3]}\end{array}$ & $\begin{array}{l}31.7(62.7)[29.2- \\
34.3]\end{array}$ \\
\hline Cycling/walking activity $(>0)$ & $\begin{array}{l}841(59.1)[56.6- \\
61.8]\end{array}$ & $\begin{array}{l}608(65.9)[62.7- \\
69.1]\end{array}$ & $\begin{array}{l}1449(61.8) \\
{[59.8-63.8]}\end{array}$ \\
\hline $\begin{array}{l}\text { Prevalence of low physical activity (Total Physical Activity MET minutes } \\
\text { per week is }<600 \text { ) }\end{array}$ & $\begin{array}{l}586(41.2)[38.8- \\
43.7]\end{array}$ & $\begin{array}{l}513(55.6)[52.1- \\
58.8]\end{array}$ & $\begin{array}{l}1099(46.9) \\
{[44.9-48.9]}\end{array}$ \\
\hline \multicolumn{4}{|l|}{ Diet } \\
\hline $\begin{array}{l}\text { Do not meet WHO recommendations ( }<5 \text { servings of fruits or vegetables } \\
\text { per day) }\end{array}$ & $\begin{array}{l}1202(84.5) \\
{[82.6-86.3]}\end{array}$ & $\begin{array}{l}839(91.0)[89.0- \\
92.7]\end{array}$ & $\begin{array}{l}2041(87.1) \\
{[85.7-88.4]}\end{array}$ \\
\hline \multicolumn{4}{|l|}{ Tobacco use } \\
\hline Currently smoke men (daily) & $\begin{array}{l}370(42.8)[39.7- \\
46.1]\end{array}$ & $\begin{array}{l}184(33.2)[29.3- \\
37.3]\end{array}$ & $\begin{array}{l}554(39.0)[36.5- \\
41.6]\end{array}$ \\
\hline Currently smoke women (daily) $\ddagger$ & - & - & $23(2.5)[1.7-3.7]$ \\
\hline Currently use smokeless tobacco men & $\begin{array}{l}139(16.1)[13.7- \\
18.6]\end{array}$ & $\begin{array}{l}248(44.7)[40.6- \\
48.9]\end{array}$ & $\begin{array}{l}387(27.3)[25.1- \\
29.5]\end{array}$ \\
\hline Currently use smokeless tobacco women & $\begin{array}{l}109(19.5)[16.3- \\
23.0]\end{array}$ & $\begin{array}{l}34(9.3)[6.4- \\
12.6]\end{array}$ & $\begin{array}{l}143(15.5)[13.2- \\
17.8]\end{array}$ \\
\hline Any form of tobacco men & $\begin{array}{l}434(50.2)[47.0- \\
53.4]\end{array}$ & $\begin{array}{l}326(58.7)[54.6- \\
63.0]\end{array}$ & $\begin{array}{l}760(53.6)[51.0- \\
56.1]\end{array}$ \\
\hline Any form of tobacco women & $\begin{array}{l}109(19.5)[16.5- \\
22.9]\end{array}$ & $\begin{array}{l}50(13.6)[10.2- \\
17.2]\end{array}$ & $\begin{array}{l}159(17.2)[14.9- \\
19.4]\end{array}$ \\
\hline \multicolumn{4}{|l|}{ Risk behaviours for infectious diseases } \\
\hline More than 2 sexual partners in the last 10 years & $\begin{array}{l}136(9.6)[8.1- \\
11.0]\end{array}$ & $\begin{array}{l}150(16.3)[13.9- \\
18.7]\end{array}$ & $\begin{array}{l}286(12.2)[10.9- \\
13.4]\end{array}$ \\
\hline \multicolumn{4}{|l|}{ Used condom when having sex in the past 10 years } \\
\hline Never & $\begin{array}{l}26(19.1)[13.1- \\
26.4]\end{array}$ & $\begin{array}{l}98(65.3)[58.3- \\
73.0]\end{array}$ & $\begin{array}{l}124(43.4)[37.5- \\
48.9]\end{array}$ \\
\hline Sometimes or always & $\begin{array}{l}110(80.9)[73.6- \\
86.9]\end{array}$ & $\begin{array}{l}52(34.6)[27.0- \\
41.7]\end{array}$ & $\begin{array}{l}162(56.6)[51.1- \\
62.5]\end{array}$ \\
\hline Ever injected street drugs, steroids, or vitamins with a needleł & - & - & $35(1.5)[1.1-2.1]$ \\
\hline \multicolumn{4}{|c|}{$\begin{array}{l}\text { * Values presented as mean (S.D.), } \neq \text { Data not reported due to low numbers for statistical disclosure control, §The denominators for the } \\
\text { HbA1c are } 2267 \text { overall, } 1370 \text { for Bangladesh, and } 897 \text { for Pakistan. \$The denominators for hypertension are } 2343 \text { overall, } 1422 \text { for } \\
\text { Bangladesh, and } 921 \text { for Pakistan. §The denominators for hypercholesterolemia are } 2247 \text { overall, } 1357 \text { for Bangladesh, and } 890 \text { for } \\
\text { Pakistan. Confidence intervals were calculated using bootstrap sampling procedure }(n=1000) \text { for binomial and continuous variables, and } \\
\text { using Goodman's method for multinomial proportions. }\end{array}$} \\
\hline
\end{tabular}


Table 3

Proportion of people with SMI screened, diagnosed and treated for NCDs and NCD risk factors, including health risk behaviour modification advice

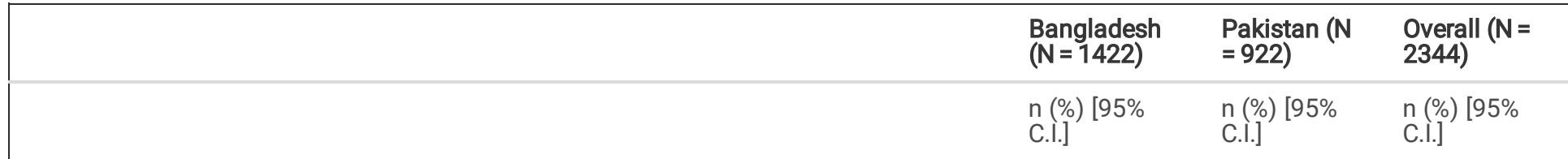

\section{Screened, diagnosed and treated for NCD and NCD risk factors (self-reported)}

Type-2 diabetes

Ever had blood glucose measured by doctor or health care provider

Ever diagnosed with type-2 diabetes by doctor or health care provider

Have received treatment for diabetes (among those with type-2 diabetes) $\S$

Unaware of having type-2 diabetes (among those with type 2 diabetes) $\S$

Hypertension

Ever had blood pressure measured by doctor or health care provider

Ever diagnosed with hypertension by doctor or health care provider

Have received treatment (among those diagnosed with hypertension) §

Unaware of having hypertension (among those diagnosed with hypertension) §

Hypercholesterolemia

Ever had cholesterol measured by doctor or health care provider

Ever diagnosed with hypercholesterolemia by

doctor or health care provider

Have received treatment (among those diagnosed with hypercholesterolemia) $\S \ddagger$

Unaware of having hypercholesterolemia (among those diagnosed with

hypercholesterolemia) $\S$

Any NCD or NCD risk factor (type II diabetes, lung disease, heart disease, hypertension

or hypercholesterolemia)

Ever been tested for any NCD or NCD risk factor 9

Ever been diagnosed with any NCD or NCD risk factor

Received treatment from doctor or other health worker for any of these (among those with NCD or NCD risk factor)

Unaware of having any NCD or NCD risk factor (among those diagnosed)

(Denominator for Bangladesh 761, Pakistan 651, Overall 1412) §

\section{Screened, diagnosed and treated for communicable diseases (self-reported)}

Ever been tested for Hepatitis B/C

Diagnosed with Hepatitis B or hepatitis C among those tested

\begin{tabular}{lll}
$317(22.3)$ & $323(35.0)$ & $640(27.3)$ \\
{$[20.0-24.5]$} & {$[32.0-38.0]$} & {$[25.6-29.1]$} \\
$38(12.0)$ & $55(17.0)$ & $93(14.5)$ \\
{$[8.7-15.8]$} & {$[13.0-20.9]$} & {$[12.0-17.5]$} \\
\hline $27(71.1)$ & $37(67.3)$ & $64(68.8)$ \\
{$[56.3-86.2]$} & {$[53.7-79.2]$} & {$[58.5-77.6]$} \\
$73(65.8)$ & $17(23.6)$ & $90(49.2)$ \\
{$[56.4-74.1]$} & {$[15.1-34.9]$} & {$[42.6-55.8]$}
\end{tabular}

$\begin{array}{lll}742(52.2) & 668(72.5) & 1410(60.2) \\ {[49.4-54.8]} & {[69.5-75.3]} & {[58.2-62.1]} \\ 82(11.1)[9.0- & 240(35.9) & 322(22.8) \\ 13.5] & {[32.2-39.5]} & {[20.8-25.0]} \\ 43(52.4) & 92(38.3) & 135(41.9) \\ {[41.8-62.5]} & {[32.1-44.7]} & {[36.6-47.6]} \\ 153(65.1) & 104(30.2) & 257(44.4) \\ {[58.8-71.0]} & {[25.6-35.3]} & {[40.6-48.2]}\end{array}$

$\begin{array}{lll}55(3.9)[3.0- & 115(12.5) & 170(7.3) \\ 4.9] & {[10.4-14.7]} & {[6.3-8.4]} \\ 13(23.6) & 45(39.1) & 58(34.1) \\ {[13.3-35.7]} & {[30.8-48.1]} & {[27.4-41.6]}\end{array}$

$\begin{array}{lll}- & - & 19(32.8) \\ & & {[21.6-46.3]} \\ 595(97.9) & 452(90.9) & 1047(94.8) \\ {[96.3-98.8]} & {[88.1-93.2]} & {[93.3-95.9]}\end{array}$

\begin{tabular}{|c|c|c|}
\hline $\begin{array}{l}786(55.3) \\
{[52.7-57.8]}\end{array}$ & $\begin{array}{l}709(76.9) \\
{[74.1-79.5]}\end{array}$ & $\begin{array}{c}1495(63.8) \\
{[61.9-65.7}\end{array}$ \\
\hline $\begin{array}{l}122(15.5) \\
{[13.2-18.2]}\end{array}$ & $\begin{array}{l}315(44.4) \\
{[40.8-48.1]}\end{array}$ & $\begin{array}{l}437(29.2) \\
{[27.1-31.5]}\end{array}$ \\
\hline $\begin{array}{l}64(52.5) \\
{[43.6-61.2]}\end{array}$ & $\begin{array}{l}128(40.6) \\
{[35.3-46.2]}\end{array}$ & $\begin{array}{l}192(43.9) \\
{[39.4-48.6]}\end{array}$ \\
\hline $\begin{array}{l}758(99.6) \\
{[98.8-99.9]}\end{array}$ & $\begin{array}{l}640(98.3) \\
{[97-99.1]}\end{array}$ & $\begin{array}{l}1398(99) \\
{[98.3-99.4]}\end{array}$ \\
\hline
\end{tabular}

\begin{tabular}{|c|c|c|c|}
\hline Ever been tested for Hepatitis B/C & $\begin{array}{l}25(1.8)[1.1- \\
2.5]\end{array}$ & $\begin{array}{l}200(21.7) \\
{[19.0-24.3]}\end{array}$ & $\begin{array}{l}225(9.6) \\
{[8.5-10.8]}\end{array}$ \\
\hline Diagnosed with Hepatitis B or hepatitis C among those tested & $\begin{array}{l}6(24.0)[11.0- \\
44.7]\end{array}$ & $\begin{array}{l}54(27.0) \\
{[20.7-33.2]}\end{array}$ & $\begin{array}{l}60(26.7) \\
{[21.2-32.9]}\end{array}$ \\
\hline
\end{tabular}




\begin{tabular}{|c|c|c|c|}
\hline & $\begin{array}{l}\text { Bangladesh } \\
(\mathrm{N}=1422)\end{array}$ & $\begin{array}{l}\text { Pakistan }(\mathrm{N} \\
=922)\end{array}$ & $\begin{array}{l}\text { Overall }(\mathrm{N}= \\
2344)\end{array}$ \\
\hline Ever been tested for tuberculosis & $\begin{array}{l}36(2.5)[1.7- \\
3.4]\end{array}$ & $\begin{array}{l}166(18.0) \\
{[15.4-20.2]}\end{array}$ & $\begin{array}{l}202(8.6) \\
{[7.6-9.8]}\end{array}$ \\
\hline Diagnosed with tuberculosis among those tested & $\begin{array}{l}18(50.0) \\
{[34.3-65.6]}\end{array}$ & $\begin{array}{l}54(32.5) \\
{[25.7-39.7]}\end{array}$ & $\begin{array}{l}72(35.6) \\
{[29.0-42.4]}\end{array}$ \\
\hline Ever been tested for HIV & $\begin{array}{l}25(1.8)[1.1- \\
2.5]\end{array}$ & $\begin{array}{l}11(1.2) \\
{[0.5-1.9]}\end{array}$ & $\begin{array}{l}36(1.5)[1.1- \\
2.1]\end{array}$ \\
\hline $\begin{array}{l}\text { Received treatment for any chronic communicable disease (among those diagnosed } \\
\text { with a chronic communicable disease) }\end{array}$ & $\begin{array}{l}55(100.0) \\
{[100.0-100.0]}\end{array}$ & $\begin{array}{l}113(91.9) \\
{[86.5-96.3]}\end{array}$ & $\begin{array}{l}168(94.4) \\
{[90.7-97.4]}\end{array}$ \\
\hline \multicolumn{4}{|l|}{ Health risk behaviour advice } \\
\hline Quit tobacco or do not start & $\begin{array}{l}118(9.6) \\
{[7.9-11.2]}\end{array}$ & $\begin{array}{l}80(15.7) \\
{[12.7-19.0]}\end{array}$ & $\begin{array}{l}198(11.4) \\
{[10.0-13.0]}\end{array}$ \\
\hline Quit tobacco among those who currently smoke or use smokeless tobacco & $\begin{array}{l}100(21.7) \\
{[18.0-25.6]}\end{array}$ & $\begin{array}{l}70(32.7) \\
{[26.3-39.1]}\end{array}$ & $\begin{array}{l}170(25.2) \\
{[22.1-28.6]}\end{array}$ \\
\hline Reduce salt in diet & $\begin{array}{l}73(6.0)[4.6- \\
7.5]\end{array}$ & $\begin{array}{l}117(22.9) \\
{[19.3-26.7]}\end{array}$ & $\begin{array}{l}190(11.0) \\
{[9.6-12.5]}\end{array}$ \\
\hline Reduce salt in diet among those diagnosed with hypertension & $\begin{array}{l}32(16.0) \\
{[11.5-21.8]}\end{array}$ & $\begin{array}{l}92(44.0) \\
{[37.4-50.9]}\end{array}$ & $\begin{array}{l}124(30.3) \\
{[26.2-34.7]}\end{array}$ \\
\hline Eat at least five servings of fruit and/or vegetables each day & $\begin{array}{l}199(16.3) \\
{[14.1-18.4]}\end{array}$ & $\begin{array}{l}193(37.8) \\
{[33.7-41.9]}\end{array}$ & $\begin{array}{l}392(22.6) \\
{[20.8-24.6]}\end{array}$ \\
\hline Reduce fat in diet & $\begin{array}{l}115(9.4) \\
{[7.8-11.2]}\end{array}$ & $\begin{array}{l}169(33.1) \\
{[29.0-37.4]}\end{array}$ & $\begin{array}{l}284(16.4) \\
{[14.7-18.3]}\end{array}$ \\
\hline Start or do more physical activity & $\begin{array}{l}142(11.6) \\
{[9.7-13.5]}\end{array}$ & $\begin{array}{l}159(31.2) \\
{[26.9-35.3]}\end{array}$ & $\begin{array}{l}301(17.4) \\
{[15.7-19.2]}\end{array}$ \\
\hline Maintain a healthy body weight or lose weight & $\begin{array}{l}95(7.8)[6.3- \\
9.3]\end{array}$ & $\begin{array}{l}91(17.8) \\
{[14.6-21.2]}\end{array}$ & $\begin{array}{l}186(10.7) \\
{[9.4-12.3]}\end{array}$ \\
\hline Maintain a healthy body weight or lose weight among those with overweight or obesity & $\begin{array}{l}49(10.6) \\
{[8.1-13.8]}\end{array}$ & $\begin{array}{l}75(25.5) \\
{[20.8-30.8]}\end{array}$ & $\begin{array}{l}124(16.4) \\
{[14.0-19.2]}\end{array}$ \\
\hline Reduce sugary beverages in diet & $\begin{array}{l}74(6.1)[4.8- \\
7.5]\end{array}$ & $\begin{array}{l}76(14.9) \\
{[11.8-18.3]}\end{array}$ & $\begin{array}{l}150(8.7) \\
{[7.4-10.1]}\end{array}$ \\
\hline Reduce sugary beverages in diet among those with type 2 diabetes & $\begin{array}{l}21(20.6) \\
{[13.8-29.7]}\end{array}$ & $\begin{array}{l}30(60.0) \\
{[45.7-72.7]}\end{array}$ & $\begin{array}{l}51(33.6) \\
{[26.9-40.9]}\end{array}$ \\
\hline Any type of lifestyle advice & $\begin{array}{l}288(23.5) \\
{[21.2-26.1]}\end{array}$ & $\begin{array}{l}319(62.5) \\
{[58.5-66.6]}\end{array}$ & $\begin{array}{l}607(35.0) \\
{[33.0-37.1]}\end{array}$ \\
\hline \multicolumn{4}{|l|}{ Health care utilization } \\
\hline Visited a doctor or other health worker in the last 12 months & $\begin{array}{l}1223(86.0) \\
{[84.3-87.7]}\end{array}$ & $\begin{array}{l}510(55.3) \\
{[52.1-58.3]}\end{array}$ & $\begin{array}{l}1733(73.9) \\
{[72.2-75.6]}\end{array}$ \\
\hline \multicolumn{4}{|c|}{$\begin{array}{l}\text { \$ people that self-reported not to have type-2 diabetes, hypertension and hypercholesterolemia or not tested but found positive with the } \\
\text { test performed during the IMPACT survey. ¥Data not reported due to low numbers for statistical disclosure control. Confidence intervals } \\
\text { were calculated using bootstrap sampling procedure }(n=1000) \text { for binomial and continuous variables, and using Goodman's method for } \\
\text { multinomial proportions. 9Includes type-2 diabetes, hypertension and hypercholesterolemia }\end{array}$} \\
\hline
\end{tabular}

Eight percent of participants had type-2 diabetes (self report of clinician diagnosis or those with HBA1c $\geq 6.5 \%$ ), $1.3 \%$ had chronic respiratory disorders and $3.6 \%$ had cardiovascular diseases, $3.1 \%$ were diagnosed with tuberculosis and $2.6 \%$ with chronic hepatitis.

Overall, $43.4 \%$ participants had overweight or obesity; most women (73.3\%) and a high proportion of men (25.6\%) had a high waist circumference. Underweight was also prevalent in $8.6 \%$ with a similar distribution in both countries.

Almost a quarter (24.7\%) either reported a diagnosis of hypertension or had high measured blood pressure ( $\geq 140 / 90 \mathrm{mmHg}), 16.5 \%$ (95\%C.I. $=14.6$ to 18.5) in Bangladesh and 37.4\% (95\%C.I. $=34.2$ to 40.4) in Pakistan. Almost half (49.2\%) were found to have hypercholesterolemia based on either previous reported diagnosis or high LDL levels; Bangladesh $(44.8 \% 95 \%$ C.I. $=42.2$ to 47.5$)$ had a lower proportion (55.8\%, 95\%C.I. 52.5 to 59.1) than Pakistan. Thirty-eight percent of participants had anaemia; this was higher in Bangladesh $(45.2 \%, 95 \%$ C.I. $=42.6$ to 48.0$)$ than in Pakistan (27.5\% 95\% C.I. $=24.7 \%$ to 30.5) (online supplemental material). Most people with 
hypercholesterolemia (94.8\%) and almost half with diabetes (49.2\%) and with hypertension (44.4\%) were previously unaware of their condition and were detected during the survey through cholesterol, $\mathrm{HbA} 1 \mathrm{c}$ and blood pressure measurements, respectively.

The majority of men (53.6\%), consumed tobacco, and 39.0\% reported smoking tobacco daily. Smoking rates in men were higher in Bangladesh $(42.8 \% 95 \%$ C.I. $=39.7$ to 46.1$)$ than in Pakistan $(33.2 \% 95 \%$ C.I. $=29.3$ to 37.3) and smokeless tobacco consumption higher in Pakistan (44.7\% 95\%C.I. $=40.6$ to 48.9) compared with Bangladesh (16.1\% 95\%C.I.=13.7 to 18.6). Twenty percent and $13.6 \%$ women also reported tobacco use in Bangladesh and Pakistan, respectively. Around half of participants did not meet the WHO recommendations for physical activity (of 600 metabolic equivalents minute/week); and $84.5 \%$ of the participants in Bangladesh, and $91.0 \%$ in Pakistan reported not to meet the WHO recommended levels of fruit and vegetable intake (at least 5 servings). Only $1.0 \%$ of males and $0.1 \%$ of females reported consuming alcohol in the last month. Less than $13 \%$ of the sample reported to have more than 2 sexual partners in the last 10 years.

As shown in Table 3, only $63.7 \%$ of the participants had been previously tested for any NCDs or NCD risk factor; $60.2 \%$ for hypertension, $27.3 \%$ for type-2 diabetes, and 7.3\% for hypercholesterolemia. In general, a low proportion of participants in both Bangladesh and Pakistan received treatment for physical conditions or to address risk factors. Of those with self-reported NCD or a NCD risk factor, only $43.5 \%$ reported receiving related treatment or health-risk modification advice. The provision of relevant treatment was highest in those reporting type-2 diabetes $(68.8 \%, 95 \%$ C.I. $=58.5$ to 77.6$)$, followed by hypertension $(41.9 \%, 95 \%$ C.I. $=36.6$ to 47.6$)$ and hypercholesterolaemia $(32.8 \%$, $95 \%$ C.I. $=21.6$ to 41.6 ). Only $35.0 \%$ received any type of advice to modify health-risk behaviours; among those who consumed tobacco, only $25.2 \%$ had been advised to quit.

\section{Comparison between people with SMI and the general population (STEPS survey)}

The results for the comparisons between our data and country STEPS reports are summarized in Table 4. 
Table 4

Odds of people with SMI having non-communicable diseases, related risk factors and health risk behaviours and receiving healthcare screening and advice compared with the general population*

\begin{tabular}{|c|c|c|c|c|c|c|c|c|c|c|}
\hline & \multirow[b]{2}{*}{$\begin{array}{l}\text { STEPS } \\
\text { (responded } \\
\text { yes/total) }\end{array}$} & \multirow[b]{2}{*}{$\begin{array}{l}\text { SMI survey } \\
\text { (responded } \\
\text { yes/total) }\end{array}$} & \multicolumn{3}{|c|}{ Bangladesh } & \multicolumn{5}{|l|}{ Pakistan } \\
\hline & & & $\begin{array}{l}\text { Odds } \\
\text { ratio }\end{array}$ & $\begin{array}{l}95 \% \\
\mathrm{Cl}\end{array}$ & $\mathrm{p} \ddagger$ & $\begin{array}{l}\text { STEPS } \\
\text { (responded } \\
\text { yes/total) }\end{array}$ & $\begin{array}{l}\text { SMI survey } \\
\text { (responded } \\
\text { yes/total) }\end{array}$ & $\begin{array}{l}\text { Odds } \\
\text { ratio }\end{array}$ & $\begin{array}{l}95 \% \\
\mathrm{Cl}\end{array}$ & $\mathrm{p} \ddagger$ \\
\hline \multicolumn{11}{|l|}{$\begin{array}{l}\text { Non-communicable } \\
\text { diseases (NCDs) }\end{array}$} \\
\hline $\begin{array}{l}\text { Type-2 diabetes } \\
\text { (diagnosed by HbA1c } \\
\text { test }(\geq 6.50 \%) \text { or self- } \\
\text { report of clinician } \\
\text { diagnosis) } \neq\end{array}$ & $586 / 7056$ & $169 / 1363$ & 1.56 & $\begin{array}{l}1.30 \\
\text { to } \\
1.88\end{array}$ & $\begin{array}{l}< \\
0.001\end{array}$ & $\mathrm{~N} / \mathrm{A}$ & $67 / 882$ & $\mathrm{~N} / \mathrm{A}$ & $\mathrm{N} / \mathrm{A}$ & $\mathrm{N} / \mathrm{A}$ \\
\hline $\begin{array}{l}\text { Type-2 diabetes (self- } \\
\text { report of clinician } \\
\text { diagnosis only) }\end{array}$ & $417 / 8185$ & $66 / 1413$ & 0.91 & $\begin{array}{l}0.69 \\
\text { to } \\
1.19\end{array}$ & 0.501 & $250 / 7358$ & $50 / 907$ & 1.66 & $\begin{array}{l}1.19 \\
\text { to } \\
2.28\end{array}$ & 0.001 \\
\hline $\begin{array}{l}\text { Cardiovascular } \\
\text { diseases (angina or a } \\
\text { stroke) (self-report of } \\
\text { clinician diagnosis) }\end{array}$ & $819 / 8185$ & $26 / 1413$ & 0.17 & $\begin{array}{l}0.11 \\
\text { to } \\
0.25\end{array}$ & $<.001$ & $363 / 7357$ & $59 / 907$ & 1.34 & $\begin{array}{l}0.99 \\
\text { to } \\
1.79\end{array}$ & 0.043 \\
\hline \multicolumn{11}{|l|}{ NCD risk factors } \\
\hline $\begin{array}{l}\text { Hypertension } \\
\text { (measured in survey or } \\
\text { self-report of clinician } \\
\text { diagnosis) }\end{array}$ & $1684 / 8019$ & $301 / 1413$ & 1.02 & $\begin{array}{l}0.89 \\
\text { to } \\
1.17\end{array}$ & 0.797 & $\mathrm{~N} / \mathrm{A}$ & $325 / 908$ & $\mathrm{~N} / \mathrm{A}$ & $\mathrm{N} / \mathrm{A}$ & $\mathrm{N} / \mathrm{A}$ \\
\hline $\begin{array}{l}\text { Hypertension (self- } \\
\text { report of clinician } \\
\text { diagnosis only) }\end{array}$ & $1121 / 8185$ & $137 / 1413$ & 0.68 & $\begin{array}{l}0.56 \\
\text { to } \\
0.82\end{array}$ & $\begin{array}{l}< \\
0.001\end{array}$ & 1096/7358 & $234 / 907$ & 1.99 & $\begin{array}{l}1.68 \\
\text { to } \\
2.34\end{array}$ & $\begin{array}{l}< \\
0.001\end{array}$ \\
\hline $\begin{array}{l}\text { Hypercholesterolemia } \\
\text { (measured in survey or } \\
\text { self-report of clinician } \\
\text { diagnosis) }\end{array}$ & 2002/7049 & $647 / 1342$ & 2.35 & $\begin{array}{l}2.08 \\
\text { to } \\
2.65\end{array}$ & $\dot{0} 0.001$ & $\mathrm{~N} / \mathrm{A}$ & $481 / 876$ & $\mathrm{~N} / \mathrm{A}$ & $\mathrm{N} / \mathrm{A}$ & $\mathrm{N} / \mathrm{A}$ \\
\hline $\begin{array}{l}\text { Hypercholesterolemia } \\
\text { (self-report of clinician } \\
\text { diagnosis only) }\end{array}$ & $176 / 8185$ & $19 / 1413$ & 0.62 & $\begin{array}{l}0.36 \\
\text { to } \\
1.00\end{array}$ & 0.048 & $110 / 7357$ & $39 / 907$ & 2.96 & $\begin{array}{l}1.99 \\
\text { to } \\
4.33\end{array}$ & $\begin{array}{l}< \\
0.001\end{array}$ \\
\hline $\begin{array}{l}\text { Underweight }(\mathrm{BMI}< \\
18 \cdot 5)\end{array}$ & $1038 / 7985$ & $112 / 1418$ & 0.57 & $\begin{array}{l}0.46 \\
\text { to } \\
0.70\end{array}$ & $\begin{array}{l}< \\
0.001\end{array}$ & $747 / 6613$ & $90 / 891$ & 0.88 & $\begin{array}{l}0.69 \\
\text { to } \\
1.11\end{array}$ & 0.285 \\
\hline $\begin{array}{l}\text { Overweight or Obesity } \\
(B M I \geq 25)\end{array}$ & $2068 / 7985$ & $576 / 1412$ & 1.97 & $\begin{array}{l}1.75 \\
\text { to } \\
2.22\end{array}$ & $<.001$ & $2725 / 6613$ & $475 / 896$ & 1.61 & $\begin{array}{l}1.40 \\
\text { to } \\
1.86\end{array}$ & $\begin{array}{l}< \\
0.001\end{array}$ \\
\hline $\begin{array}{l}\text { High waist } \\
\text { circumference women } \\
(\geq 80 \mathrm{~cm})\end{array}$ & $1687 / 4104$ & $552 / 754$ & 1.37 & $\begin{array}{l}1.10 \\
\text { to } \\
1.69\end{array}$ & 0.005 & N/A & $384 / 508$ & N/A & $\mathrm{N} / \mathrm{A}$ & $\mathrm{N} / \mathrm{A}$ \\
\hline $\begin{array}{l}\text { High waist } \\
\text { circumference men }(\geq \\
94 \mathrm{~cm})\end{array}$ & $556 / 3784$ & $126 / 660$ & 3.59 & $\begin{array}{l}3.04 \\
\text { to } \\
4.25\end{array}$ & $<.001$ & $\mathrm{~N} / \mathrm{A}$ & $167 / 384$ & $\mathrm{~N} / \mathrm{A}$ & $\mathrm{N} / \mathrm{A}$ & $\mathrm{N} / \mathrm{A}$ \\
\hline \multicolumn{11}{|l|}{ Health risk behaviours } \\
\hline $\begin{array}{l}\text { Low physical activity } \\
\text { (Total Physical Activity } \\
\text { MET minutes per week } \\
\text { is }<600 \text { ) }\end{array}$ & $999 / 8118$ & $618 / 1414$ & 5.53 & $\begin{array}{l}4.88 \\
\text { to } \\
6.26\end{array}$ & $<.001$ & $2932 / 7064$ & $549 / 907$ & 2.16 & $\begin{array}{l}1.87 \\
\text { to } \\
2.49\end{array}$ & $\begin{array}{l}< \\
0.001\end{array}$ \\
\hline $\begin{array}{l}\text { Do not meet WHO } \\
\text { recommendations (<5 } \\
\text { servings of fruits or } \\
\text { vegetables per day) }\end{array}$ & 7318/8168 & $1196 / 1413$ & 0.64 & $\begin{array}{l}0.54 \\
\text { to } \\
0.76\end{array}$ & $\begin{array}{l}< \\
0.001\end{array}$ & 6899/7339 & $820 / 907$ & 0.60 & $\begin{array}{l}0.47 \\
\text { to } \\
0.77\end{array}$ & $\begin{array}{l}< \\
0.001\end{array}$ \\
\hline
\end{tabular}




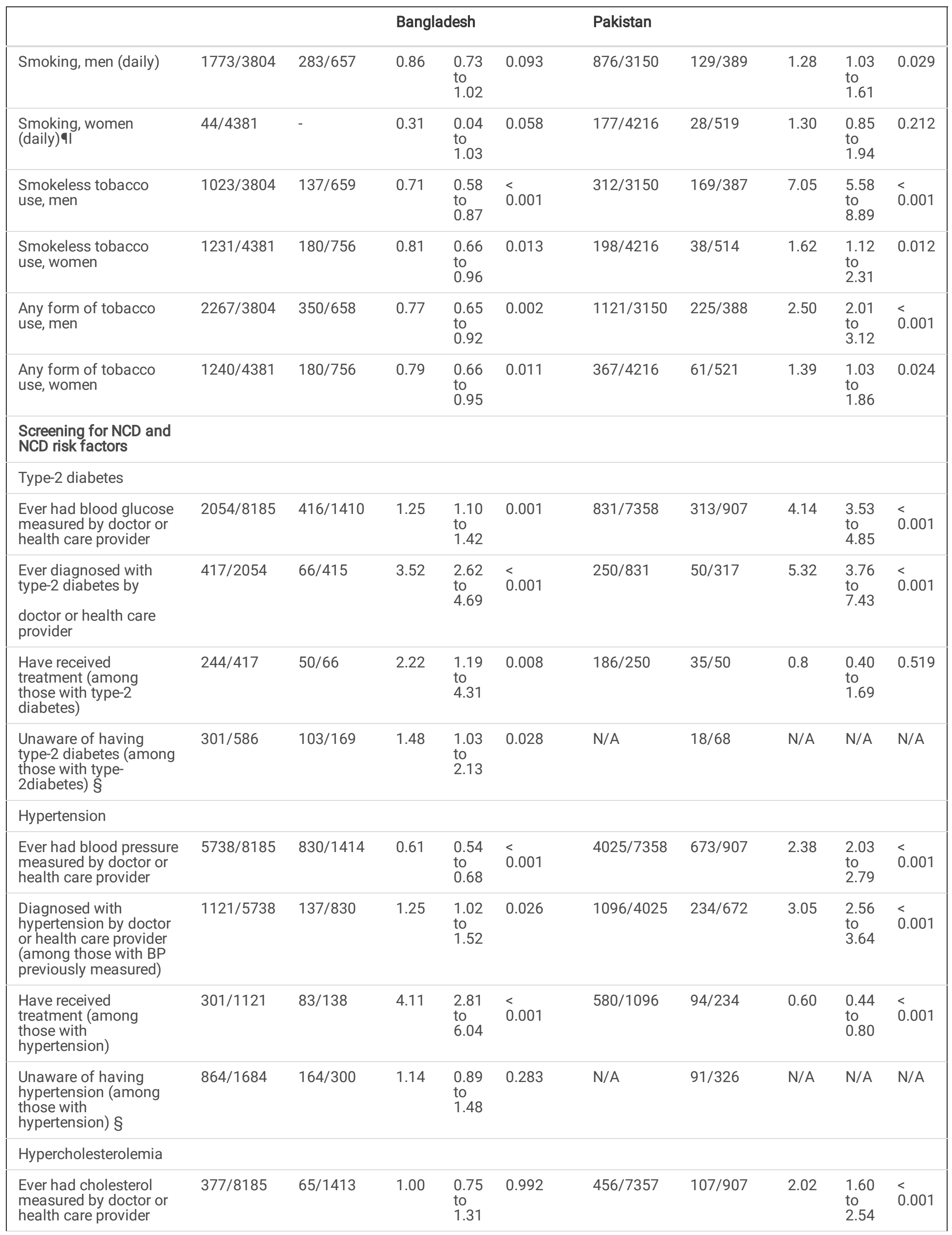




\begin{tabular}{|c|c|c|c|c|c|c|c|c|c|c|}
\hline \multirow{3}{*}{$\begin{array}{l}\text { Diagnosed with } \\
\text { hypercholesterolemia } \\
\text { by } \\
\text { doctor or health care } \\
\text { provider (among those } \\
\text { with cholesterol } \\
\text { previously measured) }\end{array}$} & \multirow{3}{*}{$176 / 377$} & \multirow{3}{*}{$19 / 67$} & \multicolumn{3}{|c|}{ Bangladesh } & \multicolumn{5}{|l|}{ Pakistan } \\
\hline & & & 0.45 & $\begin{array}{l}0.24 \\
\text { to } \\
0.82\end{array}$ & 0.005 & $110 / 456$ & $39 / 106$ & 1.83 & $\begin{array}{l}1.13 \\
\text { to } \\
2.93\end{array}$ & 0.008 \\
\hline & & & & & & & & & & \\
\hline $\begin{array}{l}\text { Have received } \\
\text { treatment (among } \\
\text { those diagnosed with } \\
\text { hypercholesterolemia)ฯ। }\end{array}$ & $71 / 176$ & - & 0.53 & $\begin{array}{l}0.14 \\
\text { to } \\
1.65\end{array}$ & 0.234 & $48 / 110$ & $14 / 39$ & 0.72 & $\begin{array}{l}0.31 \\
\text { to } \\
1.63\end{array}$ & 0.400 \\
\hline $\begin{array}{l}\text { Unaware of having } \\
\text { hypercholesterolemia } \\
\text { (among those with } \\
\text { hypercholesterolemia)§ }\end{array}$ & $1898 / 2002$ & $629 / 648$ & 1.81 & $\begin{array}{l}1.09 \\
\text { to } \\
3.16\end{array}$ & 0.017 & $\mathrm{~N} / \mathrm{A}$ & $442 / 481$ & N/A & $\mathrm{N} / \mathrm{A}$ & $\mathrm{N} / \mathrm{A}$ \\
\hline \multicolumn{11}{|l|}{$\begin{array}{l}\text { Advice on health risk } \\
\text { behaviours }\end{array}$} \\
\hline $\begin{array}{l}\text { Quit or do not take up } \\
\text { tobacco }\end{array}$ & $807 / 3977$ & $94 / 1205$ & 0.33 & $\begin{array}{l}0.26 \\
\text { to } \\
0.42\end{array}$ & $<001$ & $1832 / 7356$ & $60 / 496$ & 0.42 & $\begin{array}{l}0.31 \\
\text { to } \\
0.55\end{array}$ & $\begin{array}{l}<.001 \\
0.001\end{array}$ \\
\hline $\begin{array}{l}\text { Quit tobacco among } \\
\text { smokers }\end{array}$ & $434 / 638$ & $70 / 266$ & 0.17 & $\begin{array}{l}0.12 \\
\text { to } \\
0.23\end{array}$ & $<0.001$ & $419 / 823$ & $42 / 98$ & 0.72 & $\begin{array}{l}0.46 \\
\text { to } \\
1.13\end{array}$ & 0.132 \\
\hline $\begin{array}{l}\text { Start or do more } \\
\text { physical activity }\end{array}$ & $764 / 3977$ & $150 / 1210$ & 0.60 & $\begin{array}{l}0.49 \\
\text { to } \\
0.72\end{array}$ & $<.001$ & $2060 / 7356$ & $160 / 495$ & 1.23 & $\begin{array}{l}1.00 \\
\text { to } \\
1.50\end{array}$ & 0.041 \\
\hline $\begin{array}{l}\text { Maintain a healthy } \\
\text { body weight or lose } \\
\text { weight }\end{array}$ & $660 / 3977$ & $112 / 1217$ & 0.51 & $\begin{array}{l}0.41 \\
\text { to } \\
0.63\end{array}$ & $<.001$ & $1964 / 7356$ & $95 / 495$ & 0.65 & $\begin{array}{l}0.51 \\
\text { to } \\
0.82\end{array}$ & $\begin{array}{l}<.001 \\
0.001\end{array}$ \\
\hline \multicolumn{11}{|c|}{$\begin{array}{l}\text { *Data from the general population was extracted from the STEPS } 2018 \text { Bangladesh[15] report and STEPS } 2014 \text { Pakistan[14] report, data } \\
\text { from the SMI survey were weighted by age and gender according to the distribution of the STEPS report, N/A: Not available, } \neq \text { After } \\
\text { Bonferroni correction for multiple testing the } p<.05 \text { significance level was corrected to } p<.006 \text {. } \neq \text { Blood glucose } \geq 126 \mathrm{mg} / \mathrm{dl} \text { for the } \\
\text { STEPS survey and HbA1c } \geq 6.50 \% \text { for the SMI survey } \$ \text { People that self-reported not to have the condition or not previously tested but } \\
\text { tested positive in assessments performed for the IMPACT survey or the STEPS survey in Bangladesh. } 1 \text { I Data not reported due to low } \\
\text { numbers for statistical disclosure control. }\end{array}$} \\
\hline
\end{tabular}

Prevalence of NCDs and NCD risk factors

People with SMI in Bangladesh were more likely to have type-2 diabetes (O.R. $=1.56,95 \%$ C.I. $=1.30$ to $1.88, p<0.001)$ and hypercholesterolemia $(0 . R .=2.35,95 \%$ C.I. $=2.08$ to 2.65$)$ compared with the general population. Blood samples were not collected in the STEPS survey in Pakistan, therefore these comparisons are not available for the country.

People with SMI were more likely to be overweight or obese $(\mathrm{BMI}>25)$ compared with the general population. (O.R. $=1.94,95 \%$ C.I. to 1.75 to 2.22, $p<0.001$, Bangladesh; and O.R. $=1.61,95 \%$ C.I. to 1.39 to 1.84, $p<0.001$; Pakistan). People with SMI in Bangladesh were less likely to be underweight $(0 . R .=0.57,95 \%$ C.I. $=0.46$ to $0.70, p<0.001)$ while there were no differences in Pakistan.

Health-risk behaviours

People with SMI were more likely not to meet recommendations for physical activity (O.R. $=5.53,95 \%$ C.I. to 4.88 to $6.26, p<0.001$, Bangladesh; and O.R. $=2.16,95 \%$ C.I. to 1.87 to $2.49, p<0.001$. Pakistan), and less likely not to meet the WHO recommendations for fruit and vegetable intake (O.R. $=0.6495 \%$ C.I. to 0.54 to $0.76, p<0.001$, Bangladesh; and O.R. $=0.60,95 \%$ C.I. to 0.47 to $0.77, p<0.001$ Pakistan) .

Men with SMI in Bangladesh were less likely to use tobacco products (O.R. $=0.77,95 \%$ C.I. to 0.65 to $0.92, p=0.002)$ while the opposite was found in Pakistan (O.R. $=2.50,95 \%$ C.I. to 2.01 to $3.12, p=0.002)$.

Screening and diagnosis

People with SMI were more likely to be screened for type-2 diabetes in both Bangladesh ( $0 . R .=1.25,95 \% C . I .=1.10$ to $1.42, p=0.001)$ and Pakistan (O.R. $=4.14,95 \%$ C.I. $=3.53$ to $4.85, p<0.001)$ and those screened were also more likely to have type-2 diabetes $(0 . R .=3.52$, 
95\%C.I. $=2.62$ to $4.65, \mathrm{p}<0.001$, Bangladesh; and O.R. $=5.32,95 \%$ C.I. $=3.76$ to $7.43, p<0.001$, Pakistan).

People with SMI in Bangladesh were less likely (O.R. $=0.61,95 \%$ C.I. $=0.54$ to $0.68, p<0.001)$ to be screened for hypertension, while the opposite was found in Pakistan (O.R. $=2.38,95 \%$ C.I. $=2.03$ to $2.79, \mathrm{p}<0.001$ ). Among those screened, people with SMI in Pakistan were more likely to have hypertension $(\mathrm{OR}=3.05,95 \% \mathrm{C} . \mathrm{I} .=2.56$ to $3.64, \mathrm{p}<0.001)$ while no difference was found in Bangladesh.

Regarding hypercholesterolemia people with SMI in Pakistan were more likely to be screened (0.R. $=2.41,95 \%$ C.I. $=1.60$ to 2.54, $p<0.001)$ and those positive for the screening were more likely to have hypercholesterolemia (O.R. $=3.82,95 \%$ C.I. $=2.41$ to $6.48, p<0.001)$ than the general population. In Bangladesh there was no difference in screening; however those that were screened were less likely $(0 . R .=0.45,95 \% C . I .=0.28$ to $0.80, p=0.004)$ to have hypercholesterolemia than the general population.

Health-risk modification advice

People with SMI were less likely to receive advice to quit or not take up tobacco in both countries $(0 . R .=0.33,95 \%$.I. $=0.26$ to $0.42, p<0.001$, Bangladesh; and O.R. $=0.42,95 \%$ C.I. $=0.31$ to $0.55, p<0.001$, Pakistan). A similar pattern was observed for receiving advice on maintaining healthy body weight (O.R. $=0.51,95 \%$ C.I. $=0.41$ to $0.63, p<0.001$, Bangladesh; O.R. $=0.65,95 \%$ C.I. $=0.51$ to $0.82, p<0.001$, Pakistan).

\section{Discussion}

This is the first multi-country study from South Asia to report on physical multimorbidity, health risk behaviours and access to related healthcare in people with SMI. We found a high prevalence of physical health conditions, primarily NCDs and related risk factors. We also found that people with SMI were more likely to have NCDs and NCD risk factors (overweight/obesity, hypertension, hypercholesterolemia) and engage in some health risk behaviours (tobacco use) but less likely to receive risk modification advice than the general population. Many people with SMI in our sample reported they had never been tested or screened for NCDs or NCD risk factors despite the well-established link between SMI and cardiometabolic conditions.[4, 5] Moreover a large proportion of people with type-2 diabetes, hypertension and hypercholesterolaemia had not been previously diagnosed and these conditions were only detected on testing during the survey. Most had not received appropriate treatment and risk modification advice for their physical health. Therefore, even in the two major specialist mental health institutes included in our survey, most people with SMI failed to receive adequate screening, prevention and management of NCDs and NCDs risk factors.

The finding that people with SMI are more likely to have NCD risk factors compared with the general population extends previous findings for example for obesity,[28] hypercholesterolemia,[29] and decreased physical activity.[30] Importantly it should be noted that psychotropic medication may contribute to some of these adverse risks.[31] Almost all survey participants were prescribed antipsychotics, which are associated with tiredness and sedation, an increased risk of obesity, and adverse effects on glucose and lipid metabolism.

In Pakistan, we found a higher prevalence of tobacco use in people with SMI compared with the general population. This is consistent with other studies in people with SMI,[32] where tobacco use has been associated with a greater susceptibility to addiction because of a higher subjective experience of reward and an attempt to self-medicate to mitigate anxiety and depressive symptoms.[33, 34] Unexpectedly the opposite was found in Bangladesh. This may be because the STEPS survey for Bangladesh reported an unusually high estimate of the prevalence of tobacco use (70\% in men). The more reliable Global Adult Tobacco Survey[35] for the same period reported a prevalence of 58\% in th

e same group that is closer to the figures reported in our study. The low observed prevalence of alcohol use in both men and women is similar to the STEPS survey reports,[14, 15] and is likely to be explained on the basis of religious proscription.

Despite the high prevalences of overweight/obesity, hypercholesterolemia, hypertension and tobacco use, health-risk modification advice was provided to less than one quarter of people with SMI and we found that the odds of receiving such advice was lower in people with SMI than in the general population in both countries. Similar treatment gaps have been reported in high income countries.[36] Although psychiatrists are trained in motivational interviewing, there are attitudinal barriers that make mental health professionals reluctant to engage with patients about their tobacco use.[37] Moreover, misconceptions about potential side-effects of tobacco cessation medication, unfounded fears of exacerbating depressive symptoms following quitting and low expectations of patients' motivation or ability to stop smoking are additional barriers.[38] On the other hand, there is high quality evidence from high income countries about both the effectiveness and cost benefits of smoking cessation interventions in people with SMI.[39] Such approaches need to be adopted in South Asia, where tobacco use is common. Similarly, lifestyle interventions have shown promise in reducing weight and improving metabolic risk factors and are recommended as an essential part of the management of SMI in these countries.[36] 
An important study finding is the high proportion of participants with moderate or severe depressive and anxiety symptoms. In Pakistan this was higher than for Bangladesh, perhaps related to the higher proportion of participants in the depression with psychosis category in the Pakistan sample. Depression and anxiety are independently linked with NCDs, therefore addressing these may help to prevent and better manage NCDs in people with SMI.

The prevalence of TB was three times higher than in the general population.[40] This is consistent with previous findings and clustering of TB risk factors reported in people with SMI.[41] In contrast, the prevalence of HIV[42] and Hepatitis B and C[43] were similar to those reported in the general population, a surprising finding considering the several risk factors for blood-borne viruses that have been reported to cluster in people with SMI.[44]

While most of the comparisons between people with SMI and the general population are in line with clinical expectations and previous findings,[28-30] there were some anomalous results. These include the lower odds of people with SMI with a self-reported clinical diagnosis of type-2 diabetes, hypertension and hypercholesterolemia in Bangladesh. This may be due to 'diagnostic overshadowing' where the presence of a mental disorder means clinicians do not look for physical health problems, or failure to recall such diagnosis by patients. The lower education and socioeconomic levels for participants from Bangladesh (compared with Pakistan) may have contributed to the latter.[45, 46]

We report findings from the first large-scale effort to document physical multimorbidity in people with SMI attending specialist services in two South Asian countries. We used standardised tools for data collection (i.e. STEPS, EQ-5D, PHQ9, GAD7) that allowed us to compare our findings with those in the general population. Data were collected by trained researchers having experience of working with this population. Finally, we gathered objective data on physical conditions (including blood tests) and report on both previously diagnosed and undiagnosed conditions.

Of the several limitations that need to be mentioned, the first is that due to COVID-19 the participant sample was smaller than the originally planned sample of 1500 per site. Therefore, our findings need to be interpreted with caution. Second, while we have used findings from studies in the general population to compare and discuss our findings, caution needs to be exercised in such comparisons since the analyses were only adjusted by sex and age. Moreover, we need to be mindful of the time lag between these studies, during which a number of parameters of interest might have changed.

Third, we relied on blood results from each mental health institution's laboratory but we did not standardise these tests between laboratories. Fourth, since the sample was drawn from tertiary care, the findings may not be representative of the total SMI population in each country. However, unlike mental health services in high-income countries, tertiary care services in South Asian countries accept self-referral without the need for primary or secondary care referral and often function as 'the first port of call' for people with severe mental illness. They also attract patients from both urban and rural areas. Therefore, the study population is likely to be similar to the overall population of people with SMI in these countries.

The high prevalence of physical health conditions and health risk behaviours in SMI compared with the general population and their underdetection even in specialist centres merits attention to improve early identification, prevention and management, in line with international recommendations and guidance. There is a need to periodically audit and improve adherence to these recommendations. In view of challenging resource limitations, interventions to address health risk behaviours that are brief, and delivered by non-specialist personnel need to be tested in these settings. Integration of physical health care with mental health care that has been envisioned at all levels of mental health care delivery needs to be actioned and scaled up.[9] Representative community-based studies may further answer questions related to regional differences in physical health conditions and health risk behaviours.

\section{Conclusion}

People with SMI in South Asia have a high prevalence of NCDs, which may be due to the associated clustering of several health risk factors and behaviours in this population. There is an unmet need to address physical multimorbidity in people with SMI in South Asia. Policy makers and healthcare professionals working with people with SMI need to recognise the extent and importance of physical multimorbidity in this vulnerable group and prioritise prevention, screening and treatment of NCDs in people with SMI.

\section{Abbreviations}

WHO

World health organization

SMI

Severe mental illness 
NCD

Non-communicable diseases

STEPS

STEPwise approach to Surveillance of NCDs

LMICs

low- and middle-income countries

$\mathrm{NIMH}$

National Institute of Mental Health in Dhaka, Bangladesh; and

IOP

Institute of Psychiatry Rawalpindi, Pakistan

MINI

Mini-international neuropsychiatric interview

TB

Tuberculosis

HIV

Human immunodeficiency virus

BMI

Body Mass Index

PHQ-9

Patient Health Questionnaire

GAD-7

Generalized Anxiety Disorder-7

$\mathrm{HbA1c}$

haemoglobin, glycated haemoglobin

\section{Declarations}

\section{Ethics approval and consent to participate}

Trained researchers provided verbal and written study information to patients and their relatives or carergivers, highlighting that participation was voluntary, the decision would not affect care, and consent could be withdrawn at any stage without providing a reason. Written consent was obtained (a thumbprint was accepted where a signature could not be provided). No assessments were conducted where the patient appeared reluctant, even if consent had previously been obtained.

The study was approved by the ethics committees of the Department of Health Sciences, University of York, UK; Centre for Injury Prevention and Research, Bangladesh; and the National Bioethics Committee, Pakistan. All study procedures complied with legislation and guidance for good practice governing the participation in research of people who may lack capacity (Mental Capacity Act (UK) 2005). Participants did not receive financial inducements to participate, but results of physical health measurements and blood tests were shared with them and with the treating clinician.

\section{Consent for publication}

Not applicable

\section{Availability of data and materials}

The datasets used and/or analysed during the current study are available from the corresponding author on reasonable request.

\section{Competing interests}

The authors declare that they have no competing interests

\section{Funding}

This research was funded by the National Institute for Health Research (NIHR) (Grant: GHRG 17/63/130:) using UK aid from the UK Government to support global health research. The views expressed in this publication are those of the author(s) and not necessarily those of the NIHR or the UK Department of Health and Social Care. 
NS conceived the study. AH, AN, FA, HK, KP, KS, PM, RH and SR provided important contextual information. AH, FA, JB, KP, KS, NS and SM developed the methodology and selected the outcomes. AH, GZ and JB developed the analysis plan. AH, GZ, HK, JB and SR revised the data and conducted the statistical analysis. AH, GZ, HP, JB, KS and NS wrote the manuscript. All authors revised and approved the manuscript.

\section{Acknowledgements}

The authors would like to acknowledge Abu Musa Robin, Anjuman Jum Tithi, Tanvir Arafat, Lipon Saha, Tasmia Rahman, Dr. Md. Helaluddin, Dr. Khaleda Islam, Dr. Mamun, Dr. Bappi, from the team in Bangladesh, and Rubab Ayesha, Nida Afsheen, Najma Hayat, Zaheen Amin, Aniqa Maryam from the team in Pakistan for conducting all the interviews and physical measurements, providing technical and managerial support. The authors also would like to thank all the participants who consented and provided their time to complete the interview.

\section{References}

1. Liu NH, Daumit GL, Dua T, Aquila R, Charlson F, Cuijpers P, et al. Excess mortality in persons with severe mental disorders: a multilevel intervention framework and priorities for clinical practice, policy and research agendas. World Psychiatry. 2017;16:30-40.

2. Laursen TM. Life expectancy among persons with schizophrenia or bipolar affective disorder. Schizophr Res. 2011;131:101-4.

3. Mai Q, Holman CD, Sanfilippo FM, Emery JD. The impact of mental illness on potentially preventable hospitalisations: a populationbased cohort study. BMC Psychiatry. 2011;11:163.

4. Fekadu A, Medhin G, Kebede D, Alem A, Cleare AJ, Prince M, et al. Excess mortality in severe mental illness: 10-year population-based cohort study in rural Ethiopia. Br J Psychiatry. 2015;206:289-96.

5. Teferra S, Shibre T, Fekadu A, Medhin G, Wakwoya A, Alem A, et al. Five-year mortality in a cohort of people with schizophrenia in Ethiopia. BMC Psychiatry. 2011;11:165.

6. Nambiar D, Razzak J, Afsana K, Adams AM, Hasan A, Mohan D, et al. Mental illness and injuries: emerging health challenges of urbanisation in South Asia. BMJ. 2017;357:j1126.

7. Misra A, Tandon N, Ebrahim S, Sattar N, Alam D, Shrivastava U, et al. Diabetes, cardiovascular disease, and chronic kidney disease in South Asia: current status and future directions. BMJ. 2017;357:j1420.

8. Shavitt S, Cho YI, Johnson TP, Jiang D, Holbrook A, Stavrakantonaki M. Culture moderates the relation between perceived stress, social support, and mental and physical health. J Cross Cult Psychol. 2016;47:956-80.

9. The, Lancet. Making more of multimorbidity: an emerging priority. Lancet. 2018;391:1637.

10. UN. Transforming our world: the 2030 Agenda for Sustainable Development. New York: United Nations; 2015.

11. G. A. Zavala, K. Prasad, F. Aslam, D. Barua, A. Haidar C. Hewitt, R. Huque, S. Mansoor, P. Murthy, A. T. Nizami, N. Siddiqi, S. Sikander, K. Siddiqi, J. R. Boehnke on behalf of the IMPACT team. Prevalence of physical health conditions and health risk behaviours in people with severe mental IIIness in South Asia: Protocol for a cross-sectional study (IMPACT SMI survey). BMJ Open. 2020. doi:bmjopen-2020037869.

12. Lecrubier Y, Sheehan DV, Weiller E, Amorim P, Bonora I, Harnett Sheehan K, et al. The Mini International Neuropsychiatric Interview (MINI). A short diagnostic structured interview: reliability and validity according to the CIDI. Eur Psychiatry. 1997;12:224-31.

13. Bonita R, Winkelmann R, Douglas KA, de Courten M. The WHO Stepwise approach to surveillance (STEPS) of non-communicable disease risk factors. In: Global behavioral risk factor surveillance. Springer; 2003. p. 9-22.

14. Rafique I, Saqib MAN, Munir MA, Qureshi H, Rizwanullah KSA, Khan SA, et al. Prevalence of risk factors for noncommunicable diseases in adults: key findings from the Pakistan STEPS survey. East Mediterr Health J. 2018;24:33-41.

15. World Health Organization, Others. National STEPS survey for non-communicable diseases risk factors in Bangladesh 2018.2018. https://apps.who.int/iris/bitstream/handle/10665/332886/STEPS-BAN-eng.pdf.

16. World Health Organization. Noncommunicable Diseases, Mental Health Cluster. WHO STEPS surveillance manual : the WHO STEPwise approach to chronic disease risk factor surveillance / Noncommunicable Diseases and Mental Health, World Health Organization. World Health Organization; 2005. https://apps.who.int/iris/handle/10665/43376. Accessed 9 May 2019.

17. Bazzano LA, Joint FAO, Organization WH, Others. Dietary intake of fruit and vegetables and risk of diabetes mellitus and cardiovascular diseases [electronic resource]. World Health Organization; 2005.

18. Cleland CL, Hunter RF, Kee F, Cupples ME, Sallis JF, Tully MA. Validity of the global physical activity questionnaire (GPAQ) in assessing levels and change in moderate-vigorous physical activity and sedentary behaviour. BMC Public Health. 2014;14:1255. 
19. Gerbert B, Bronstone A, McPhee S, Pantilat S, Allerton M. Development and testing of an HIV-risk screening instrument for use in health care settings. Am J Prev Med. 1998;15:103-13.

20. Chandra PS, Carey MP, Carey KB, Prasada Rao PSDV, Jairam KR, Thomas T. HIV risk behaviour among psychiatric inpatients: results from a hospital-wide screening study in southern India. Int J STD AIDS. 2003;14:532-8.

21. Kroenke K, Spitzer RL. The PHQ-9: a new depression diagnostic and severity measure. Psychiatr Ann. 2002;32:509-15.

22. Löwe B, Decker O, Müller S, Brähler E, Schellberg D, Herzog W, et al. Validation and standardization of the Generalized Anxiety Disorder Screener (GAD-7) in the general population. Med Care. 2008;46:266-74.

23. EuroQol Group,. EQ-5D-5L User Guide: Basic information on how to use the EQ-5D-5L instrument (version 2.0) 2013 04/03/2015. 1990. http://www.euroqol.org/fileadmin/user_upload/Documenten/PDF/Folders_Flyers/UserGuide_EQ-5D-5L_v2.0_October_2013.pdf.

24. Lorenzo C, Williams K, Hunt KJ, Haffner SM. ... Panel III, International Diabetes Federation, and World Health Organization definitions of the metabolic syndrome as predictors of incident cardiovascular disease and .... Diabetes Care. 2007.

https://care.diabetesjournals.org/content/30/1/8?

utm_source=TrendMD\&utm_medium=cpc\&utm_campaign=Diabetes_Care_TrendMD_0.

25. World Health Organization, Others. Global status report on noncommunicable diseases 2014. World Health Organization; 2014.

26. World Health Organization. Iron deficiency anemia. Assessment, prevention, and control. A guide for programme managers. $2001 ;: 47-62$.

27. Aickin M, Gensler H. Adjusting for multiple testing when reporting research results: the Bonferroni vs Holm methods. Am J Public Health. 1996;86:726-8.

28. Bradshaw T, Mairs H. Obesity and Serious Mental III Health: A Critical Review of the Literature. Healthcare (Basel). 2014;2:166-82.

29. Vancampfort D, Stubbs B, Mitchell AJ, De Hert M, Wampers M, Ward PB, et al. Risk of metabolic syndrome and its components in people with schizophrenia and related psychotic disorders, bipolar disorder and major depressive disorder: a systematic review and metaanalysis. World Psychiatry. 2015;14:339-47.

30. Soundy A, Wampers M, Probst M, De Hert M, Stubbs B, Vancampfort D, et al. Physical activity and sedentary behaviour in outpatients with schizophrenia: A systematic review and meta-analysis. Int J Ther Rehabil. 2013;20:588-95.

31. Correll CU, Detraux J, De Lepeleire J. Effects of antipsychotics, antidepressants and mood stabilizers on risk for physical diseases in people with schizophrenia, depression and bipolar disorder. WORLD. 2015. https://onlinelibrary.wiley.com/doi/abs/10.1002/wps.20204.

32. Evins AE, Cather C, Laffer A. Treatment of tobacco use disorders in smokers with serious mental illness: toward clinical best practices. Harv Rev Psychiatry. 2015;23:90-8.

33. Prochaska JJ. Smoking and mental illness-breaking the link. N Engl J Med. 2011;365:196-8.

34. Peckham E, Bradshaw TJ, Brabyn S, Knowles S, Gilbody S. Exploring why people with SMI smoke and why they may want to quit: baseline data from the SCIMITAR RCT. J Psychiatr Ment Health Nurs. 2016;23:282-9.

35. World Health Organization, Others. Global Adult Tobacco Survey ( GATS): Bangladesh Factsheet 2009. 2009. https://apps.who.int/iris/bitstream/handle/10665/331143/ban_gats_fs_2009-eng.pdf.

36. Sheals K, Tombor I, McNeill A, Shahab L. A mixed-method systematic review and meta-analysis of mental health professionals' attitudes toward smoking and smoking cessation among people with mental illnesses. Addiction. 2016;111:1536-53.

37. Rogers ES, Gillespie C, Smelson D, Sherman SE. A Qualitative Evaluation of Mental Health Clinic Staff Perceptions of Barriers and Facilitators to Treating Tobacco Use. Nicotine Tob Res. 2018;20:1223-30.

38. Kerr S, Woods C, Knussen C, Watson H, Hunter R. Breaking the habit: a qualitative exploration of barriers and facilitators to smoking cessation in people with enduring mental health problems. BMC Public Health. 2013. https://link.springer.com/article/10.1186/14712458-13-221.

39. Gilbody S, Peckham E, Bailey D, Arundel C, Heron P, Crosland S, et al. Smoking cessation for people with severe mental illness (SCIMITAR+): a pragmatic randomised controlled trial. The Lancet Psychiatry. 2019;6:379-90.

40. Fatima Jehangir, Rizwana Hashmi, Tooba Khalid Khan Alizay Lateef, Maha Zubair, Abeer Tasleem, Arwa Giani, Ahmed Ghaffar and Jawed Usman. Prevalence and Outcomes of Tuberculosis Treatment in a Primary Care Center in Karachi, Pakistan. Archives of Medicine. 2020;12. doi:10.36648/1989-5216.12.6.337.

41. Doherty AM, Kelly J, McDonald C, O’Dywer AM, Keane J, Cooney J. A review of the interplay between tuberculosis and mental health. Gen Hosp Psychiatry. 2013;35:398-406.

42. Khan AA, Khan A. The HIV epidemic in Pakistan. J Pak Med Assoc. 2010;60:300-7.

43. Ali SA, Donahue RMJ, Qureshi H, Vermund SH. Hepatitis B and hepatitis C in Pakistan: prevalence and risk factors. Int J Infect Dis. 2009;13:9-19. 
44. Hughes E, Bassi S, Gilbody S, Bland M, Martin F. Prevalence of HIV, hepatitis B, and hepatitis C in people with severe mental illness: a systematic review and meta-analysis. Lancet Psychiatry. 2016;3:40-8.

45. Vellakkal S, Subramanian SV, Millett C, Basu S, Stuckler D, Ebrahim S. Socioeconomic Inequalities in Non-Communicable Diseases Prevalence in India: Disparities between Self-Reported Diagnoses and Standardized Measures. PLoS ONE. 2013;8:e68219. doi:10.1371/journal.pone.0068219.

46. Hosseinpoor AR, Bergen N, Mendis S, Harper S, Verdes E, Kunst A, et al. Socioeconomic inequality in the prevalence of noncommunicable diseases in low- and middle-income countries: results from the World Health Survey. BMC Public Health. 2012;12:474.

\section{Figures}

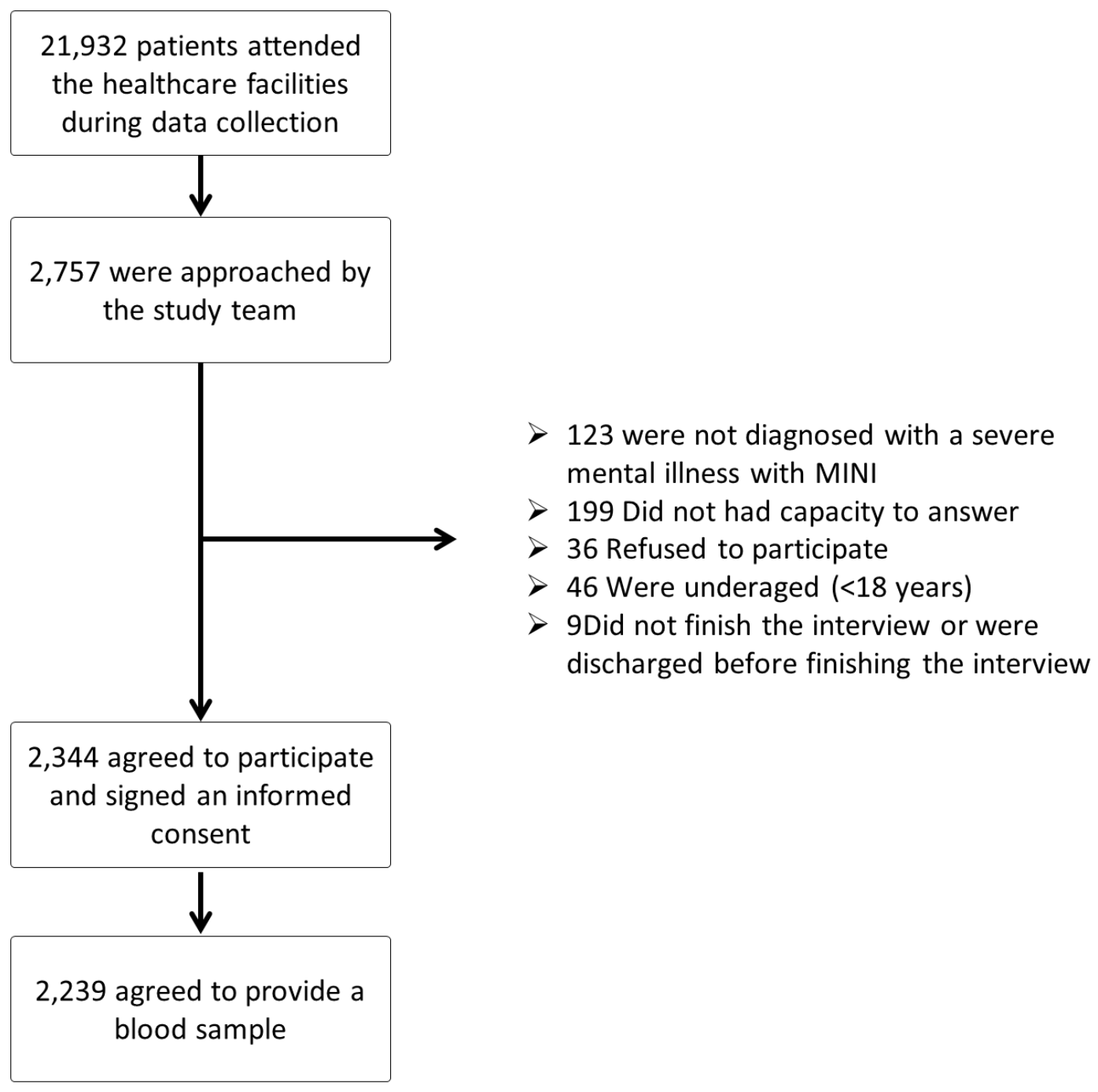

Figure 1

Between July 2019 and March 2020, 2,757 people with SMI were approached in the two sites and 2,344 (85.0\%) participated in the survey (1422 in Bangladesh and 922 in Pakistan). Out of those who did not participate, 368 were ineligible, 36 refused and 9 left before completing the survey (figure 1).

\section{Supplementary Files}

This is a list of supplementary files associated with this preprint. Click to download. 
- Appendix.docx

Page 22/22 